\title{
Article
}

\section{Time-Like Proton Form Factors in Initial State Radiation Process}

\author{
Dexu Lin $1, a * \mathbb{C}$, Alaa Dbeyssi ${ }^{2 *}$ and Frank Maas $2, b, c *$ (I) \\ 1 Institute of Modern Physics, Chinese Academy of Sciences, Nanchang Rd. 509, Lanzhou 730000, China \\ 2 Helmholtz Institute Mainz, Johann-Joachim-Becher-Weg 45, D-55099 Mainz, Germany; GSI \\ Helmholtzzentrum für Schwerionenforschung GmbH, 64291 Darmstadt, Germany \\ a University of Chinese Academy of Sciences, Beijing 100049, China \\ $b$ Institute of Nuclear Physics, Johannes Gutenberg University, Mainz, Germany \\ c Prisma Cluster of Excellence, Mainz, Germany \\ * Correspondence: dxlin@impcas.ac.cn (D.L.); adbeyssi@uni-mainz.de (A.D.) and maas@him.uni-mainz.de \\ (F.M.)
}

Abstract: Electromagnetic form factors are fundamental quantities describing the internal struc-
ture of hadrons. They can be measured with scattering processes in the space-like region and
annihilation processes in the time-like region. The two regions are connected by crossing symmetry.
The measurements of the proton electromagnetic form factors in the time-like region using the
initial state radiation technique are reviewed. Recent experimental studies have shown that initial
state radiation processes at high luminosity electron-positron colliders can be effectively used to
probe the electromagnetic structure of hadrons. The BABAR experiment at the $B$-factory PEP-II
in Stanford and the BESIII experiment at the $\tau$-charm factory BEPCII in Beijing have measured
the time-like form factors of the proton using the initial state radiation process $e^{+} e^{-} \rightarrow p \bar{p} \gamma$. The
two kinematical regions where the photon is emitted from the initial state at small and large polar
angles have been investigated. In the first case the photon is in the region not covered by the
detector acceptance and is not detected. The Born cross section and the proton effective form factor
have been measured over a wide and continuous range of the the momentum transfer squared $q^{2}$
from threshold up to 42 (GeV/c) $)^{2}$. The ratio of electric and magnetic form factors of the proton
has been also determined. In this report, the theoretical aspect and the experimental studies of
the initial state radiation process $e^{+} e^{-} \rightarrow p \bar{p} \gamma$ are described. The measurements of the Born cross
section and the proton form factors obtained in these analyses near the threshold region and in the
relatively large $q^{2}$ region are examined. The experimental results are compared to the predictions
from theory and models. Their impact on our understanding of the nucleon structure is discussed.

Keywords: proton, electromagnetic form factors, initial state radiation, time-like

\section{Introduction}

The concept of symmetry plays a key role for understanding the fundamental building blocks of the universe and the forces binding them. Experiments employing antimatter, a mirror or a counterpart of a subatomic particle with opposite charge and right- or left-handed spin, provide important information about the elementary units of which the universe is composed. Measurements of the exclusive production of hadron pair in electron positron annihilation for example are of great interest for understanding the internal structure of hadrons. Assuming the exchange of a virtual photon of positive momentum transfer squared, $q^{2}$, between the initial and the final states, these processes contain direct information on the electromagnetic form factors (EMFFs) in the time-like region. The number of independent EMFFs is determined by the spin of the hadron taking into account symmetry properties of the electromagnetic interaction under the Charge, Parity, and Time (CPT) transformations. Spin $1 / 2$ baryons are characterized by the electric form factor $G_{E}\left(q^{2}\right)$ and magnetic form factor $G_{M}\left(q^{2}\right)$. In the space-like region $\left(q^{2}<0\right)$, the EMFFs for stable baryons are experimentally accessible 
through the measurements of cross sections for elastic electron baryon scattering. The crossed symmetry channels $e^{+} e^{-} \leftrightarrow B \bar{B}$ allow to access the Time-Like region. Crossing symmetry, which holds at the tree level, states that the same amplitude describes the crossed processes, which occur in different kinematical regions, and connect the timelike with the space-Like EMFFs. In the $e^{+} e^{-}$experiments, time-like EMFFs have been measured using the so-called energy scan technique, in which the center of mass (c.m.) energy $(\sqrt{s})$ of the collider is varied systematically, and at each c.m. energy point a measurement of the associated cross section is carried out through the $e^{+} e^{-}$direct annihilation process. This method, however, can not be used at $e^{+} e^{-}$colliders that are designed to operate at fixed c.m. energies such as the B-factories PEP-II at SLAC and KEK-B in Tsukuba with a $\sqrt{s}$ corresponding to the mass of the $\mathrm{Y}(4 S)$-resonance. A complementary approach to the energy scan technique that allows the measurements of the hadronic final states with invariant masses below the c.m. energy of the collider is provided by the initial state radiation (ISR) method. The ISR method, also called radiative return technique, employs measurements the process $e^{+} e^{-} \rightarrow$ hadrons $+n \gamma$ where the emission of one or more high energy photons reduces the mass squared of the hadronic system to below $\sqrt{s}$.

The theoretical studies of the ISR processes started long time ago in the sixties of the $20^{\text {th }}$ century. The importance of the ISR approach has been outlined in the calculation of the photon emission for muon pair production in Ref. [1] and for pions in Ref. [2]. The possibility of using this approach at the high luminosity $e^{+} e^{-}$colliders has been discussed later [3-6], in particular when the first generation of $\Phi$ and $B$ factories came in operation in the late nineties. It has been realized that the suppression factor $\alpha / \pi$, where $\alpha$ is the fine electromagnetic constant, due to the photon emission can be well compensated by the high luminosities collected at the $e^{+} e^{-}$facilities, providing precise measurements of hadronic final states over a wide c.m. energy range. In addition, this method provides a great control of systematic effects since only one experimental setup is involved in this wide range coverage.

The ISR approach became a powerful tool for the analysis of experiments after the development of dedicated Monte Carlo event generators for the ISR processes, including higher order QED corrections, such as the PHOKHARA event generator $[7,8]$ and the AFKQED package (based on an early version of PHOKHARA called EVA [5,9]). The theoretical accuracy and the flexibility provided by these event generators have allowed experiments like BABAR, Belle, KLOE and BESIII to simulate a large number of hadronic reactions and perform precise measurements of the corresponding cross sections.

In the time-like region, Measurements of baryon EMFFs using the ISR technique have been provided by BABAR, Belle and BESIII experiments. The BABAR experiment at PEP-II [10] has measured the time-like EMFFs of the proton with the ISR process $e^{+} e^{-} \rightarrow p \bar{p} \gamma$ [11-13] using data sets of integrated luminosity up to $469 \mathrm{fb}^{-1}$. The two kinematical regions where the photon is emitted from the initial state at small and large polar angles have been investigated. In the first case, the small angle ISR (SA-ISR, also called ISR-Untagged), the photon is in the region not covered by the detector acceptance and is not detected, while in the second case for the large angle ISR (LA-ISR, also called ISR-Tagged), the photon has to be detected and all the particles in the final state are fully reconstructed. The Born cross section and the proton effective form factor have been measured over a wide and continuous range of the the momentum transfer squared $q^{2}$ from threshold up to $42(\mathrm{GeV} / \mathrm{c})^{2}$ considering both analyses. The ratio of electric and magnetic form factors of the proton has been also determined in the LA-ISR analysis. The hyperon final states $\Lambda \bar{\Lambda} \gamma, \Lambda \bar{\Sigma}^{0} \gamma, \Sigma \bar{\Sigma}^{0} \gamma$ have been also investigated by BABAR [14]. The $\left|G_{E}\right| /\left|G_{m}\right|$ ratio for the $\Lambda$ hyperon has been measured in two mass intervals between 2.23 and $2.80 \mathrm{GeV} / \mathrm{c}^{2}$. The Belle collaboration at KEK-B used the ISR-technique in the charmonium energy region and measured the process $e^{+} e^{-} \rightarrow \Lambda_{c}^{+} \Lambda_{c}^{-}$[15]. The BESIII experiment in Beijing provided the world's largest samples of $e^{+} e^{-}$collisions in the $\tau$ charm region. Large data samples have been accumulated at c.m. energies corresponding 
to the charmonium and $X, Y$ and $Z$ states. At $\sqrt{s}=3.773 \mathrm{GeV}$, an integrated luminosity of $2.9 \mathrm{fb}^{-1}$ has been accumulated and used for most of the ISR analyses. Using these data samples, precise measurements of the proton EMFFs have been performed with the ISR method [16,17]. Both LA- and SA-ISR approaches have been studied at BESIII.

Important results with the ISR technique have been also obtained for mesonic final states. Precise measurements, for example, on the cross section for the processes $e^{+} e^{-} \rightarrow \pi^{+} \pi^{-}$and $e^{+} e^{-} \rightarrow K^{+} K^{-}$have been provided by KLEO, BABAR, and BESIII experiments [18-25]. These measurements cover a wide mass range and have a large impact on the calculation of the hadronic contribution to the anomalous magnetic moment of the muon.

In this report, we describe the ISR analyses for the proton EMFFs measurements at BABAR and BESIII experiments. In section 2, the theoretical calculations of the differential and integrated cross section for the process $e^{+} e^{-} \rightarrow p \bar{p} \gamma$ are described. The experimental results of the proton EMFFs measurements from both experiments are reviewed in section 3 , and following by a discution in section 4 .

\section{Theoretical description for the process of $e^{+} e^{-} \rightarrow p \bar{p} \gamma$}

2.1. Born cross section for $e^{+} e^{-} \rightarrow p \bar{p}$ process

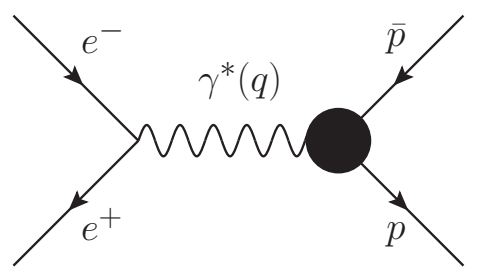

Figure 1. Feynman diagram for the process of $e^{+} e^{-} \rightarrow p \bar{p}$ under the assumption of one virtual photon exchange. The bold vertex provides access to the hadronic FFs information.

In the $e^{+} e^{-}$c.m. frame, the differential cross section for the process of $e^{+} e^{-} \rightarrow p \bar{p}$ under the assumption of one virtual photon exchange (Fig. 1) is [26],

$$
\frac{d \sigma_{p \bar{p}}\left(q^{2}\right)}{d \cos \vartheta_{p}}=\frac{\pi \alpha^{2} \beta \mathcal{C}}{2 q^{2}}\left[\left|G_{\mathrm{M}}\left(q^{2}\right)\right|^{2}\left(1+\cos ^{2} \vartheta_{p}\right)+\frac{\left|G_{\mathrm{E}}\left(q^{2}\right)\right|^{2}}{\tau} \sin ^{2} \vartheta_{p}\right],
$$

where $q^{2}$ is equal to the square of the $p \bar{p}$ invariant mass $M_{p \bar{p}}, \alpha \approx \frac{1}{137}$ is the fine structure constant, $\beta=\sqrt{1-1 / \tau}$ is the velocity of the proton with $\tau=q^{2} / 4 m_{p}^{2}$ and $m_{p}$ the proton mass, and $\vartheta_{p}$ is the polar angle of the proton in the $e^{+} e^{-}$c.m. frame where the z-axis points along the direction of the positron momentum. The Coulomb factor $\mathcal{C}=\frac{y}{1-e^{-y}}$ with $y=\frac{\pi \alpha}{\beta}$ accounts for the electromagnetic interaction between the outgoing proton and antiproton $[12,27]$. The cross section depends on the moduli of the magnetic and electric FFs, which can be determined from the analysis of the proton angular distribution. By integrating the differential cross section (Eq. (1)), the total cross section for the process $e^{+} e^{-} \rightarrow p \bar{p}$ is obtained,

$$
\sigma_{p \bar{p}}\left(q^{2}\right)=\frac{4 \pi \alpha^{2} \beta \mathcal{C}}{3 q^{2}}\left[\left|G_{M}\right|^{2}+\frac{\left|G_{E}\right|^{2}}{2 \tau}\right] .
$$

An effective form factor can be introduced as a combination of $\left|G_{M}\right|^{2}$ and $\left|G_{E}\right|^{2}$,

$$
\left|G_{\text {eff }}\right|=\sqrt{\frac{2 \tau\left|G_{M}\right|^{2}+\left|G_{E}\right|^{2}}{2 \tau+1}},
$$

which is equivalent to $\left|G_{M}\right|$ determined from Eq. 2 under the assumption of $\left|G_{M}\right|=\left|G_{E}\right|$. 


\subsection{Cross section for the $e^{+} e^{-} \rightarrow p \bar{p} \gamma$ process}

The cross section for the lowest order ISR process shown in Fig. 2, i.e. including only one photon emission from the initial state and integrated over the proton momenta, can be written as $[5,11,28]$,

$$
\begin{aligned}
\frac{d \sigma_{p \bar{p} \gamma}\left(q^{2}\right)}{d q^{2} d \cos \theta_{\gamma}^{*}} & =\frac{1}{s} W\left(s, x, \theta_{\gamma}^{*}\right) \sigma_{p \bar{p}}\left(q^{2}\right), \\
x & =\frac{2 E_{\gamma}^{*}}{\sqrt{s}}=1-\frac{q^{2}}{s},
\end{aligned}
$$

where $E_{\gamma}^{*}$ and $\theta_{\gamma}^{*}$ are the energy and the polar angle of the ISR photon, respectively, in the $e^{+} e^{-}$c.m. frame. The radiator function $W\left(s, x, \theta_{\gamma}^{*}\right)$ which describes the probability of ISR photon emission, is written as follows $[2,28,29]$,

$$
\begin{aligned}
W\left(s, x, \theta_{\gamma}^{*}\right)= & \frac{\alpha}{\pi x}\left[\frac{\left(1-x+x^{2} / 2\right) \sin ^{2} \theta_{\gamma}^{*}-\left(x^{2} / 2\right) \sin ^{4} \theta_{\gamma}^{*}}{\left(\sin ^{2} \theta_{\gamma}^{*}+\left(4 m_{e}^{2} / s\right) \cos ^{2} \theta_{\gamma}^{*}\right)^{2}}\right. \\
& \left.-\frac{4 m_{e}^{2}}{s} \frac{(1-2 x) \sin ^{2} \theta_{\gamma}^{*}-x^{2} \cos ^{4} \theta_{\gamma}^{*}}{\left(\sin ^{2} \theta_{\gamma}^{*}+\left(4 m_{e}^{2} / s\right) \cos ^{2} \theta_{\gamma}^{*}\right)^{2}}\right],
\end{aligned}
$$

where $m_{e}$ is the electron mass. The integration of $W\left(s, x, \theta_{\gamma}^{*}\right)$ over the ISR photon angle is given for two practically cases [28,29]. The first one is given for a range of integration $\theta_{\gamma}^{0}<\theta_{\gamma}^{*}<\left(\pi-\theta_{\gamma}^{0}\right)$ and $\theta_{\gamma}^{0} \gg m_{e} / \sqrt{s}$,

$$
W(s, x)=\frac{\alpha}{\pi x}\left[\left(2-2 x+x^{2}\right) \ln \frac{1+\cos \theta_{\gamma}^{0}}{1-\cos \theta_{\gamma}^{0}}-x^{2} \cos \theta_{\gamma}^{0}\right] .
$$

For the full range of the ISR polar angle $\left(\theta_{\gamma}^{0}=0\right)$,

$$
W(s, x)=\frac{\alpha}{\pi x}\left(\ln \frac{s}{m_{e}^{2}}-1\right)\left(2-2 x+x^{2}\right) .
$$

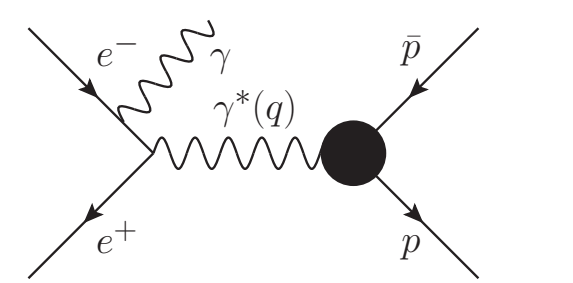

Figure 2. Lowest order Feynman diagram of the process $e^{+} e^{-} \rightarrow p \bar{p} \gamma$ assuming one virtual photon exchange, where $\gamma$ is a real photon emitted from the initial state.

The measurement of the proton EMFFs $\left|G_{E}\right|,\left|G_{M}\right|$ and their ratio $\left(R_{\mathrm{em}}=\left|G_{E}\right| /\left|G_{M}\right|\right)$ using the process $e^{+} e^{-} \rightarrow p \bar{p} \gamma$ can be performed by studying the angular distribution of the proton. BABAR and BESIII experiments measured the ratio $R_{\mathrm{em}}$ by analyzing the $\cos \theta_{p}$ distributions, where $\theta_{p}$ is the angle between the proton in the $p \bar{p}$ rest system and the momentum of the $p \bar{p}$ system in the $e^{+} e^{-}$c.m. frame.

\subsection{Radiative corrections on the $e^{+} e^{-} \rightarrow p \bar{p} \gamma$ process}

The emission of extra photons from the charged particles involved in the reaction $e^{+} e^{-} \rightarrow p \bar{p} \gamma$ requires precise calculation of the differential cross section at higher orders in the expansion of the fine structure constant $\alpha$. Radiative corrections are usually incorporated in event generators and applied to the experimental analyses taking into account the conditions of these experiments. This procedure allows for the convolution 
between the radiative corrections and the corrections of other experimental factors like for example the detection efficiency and the acceptance.

In the simulations of the $e^{+} e^{-} \rightarrow p \bar{p} \gamma$ process at large photon emission angle, the BABAR collaboration has considered the additional photon radiation from the initial state using the structure function method [31,32]. This correction takes into account soft multi-photon emission and $\alpha^{2}$ terms in the leading logarithmic calculations. The radiative correction factor calculated by BABAR includes initial state radiation and the effect of loops at the electron vertex, but does not include corrections for leptonic and hadronic vacuum polarization in the photon propagator.

The BESIII collaboration used the radiative correction calculations provided by the PHOKHARA event generator. The full next-to-leading (NLO) order QED corrections to ISR in the process $e^{+}+e^{-} \rightarrow \gamma+$ hadrons, where $\gamma$ is emitted at non vanishing angle relative to the electron or positron beams, is considered in Ref. [8]. This includes virtual and soft photon corrections to the process $e^{+}+e^{-} \rightarrow \gamma+$ hadrons and the emission of two real hard photons $e^{+}+e^{-} \rightarrow \gamma+\gamma+$ hadrons. These calculations have been extended in Refs. $[33,34]$ to the collinear region where the photons can be emitted at large and small angles relative to the electron or positron beams. The contrbution of the leptonic and hadronic vacuum polarization in the virtual photon line has also been taken into account [35]. The influence of final state radiation has been investigated in Ref. [36].

\section{Experimental results of proton form factors from ISR process}

The proton EMFFs have been measured through the ISR technique at BABAR and BESIII experiments, with both SA- and LA-ISR photon kinematic ranges.

\subsection{ISR process $e^{+} e^{-} \rightarrow p \bar{p} \gamma$ at BABAR and BESIII experiments}

The BABAR experiment started to take physics data in October 1999 and completed data taking in April 2008. Analyses through the ISR process were performed for the full data ${ }^{1}$ with an integrated luminosity of $469 \mathrm{fb}^{-1}$, which were collected at an c.m. energy of $10.58 \mathrm{GeV}(\mathrm{Y}(4 \mathrm{~S}))$ and $10.54 \mathrm{GeV}$ for about $90 \%$ and $10 \%$, respectively $[12,13]$. Depending on whether the ISR photon is detected within the detection volume or escapes through the beam pipes, the analyses are referred to as LA-ISR or SA-ISR, respectively. The event selection for both types of analyses requires that two charged tracks originating from the interaction region are detected and identified as a proton or antiproton. For the LA-ISR analysis, additional photons have to be reconstructed in the events and a four-momentum kinematic fit is applied for the proton, antiproton and most-energetic photon. For the SA-ISR analysis, a requirement on $p \bar{p}$ transverse momentum is applied to remove the LA-ISR events and to suppress the background events. Figure 3 shows the $p \bar{p}$ invariant mass distribution from the LA-ISR and SA-ISR analyses.

1 BABAR experiment has performed the LA-ISR analysis with half of luminosity, $232 \mathrm{fb}^{-1}$, in 2006 [11]. In this report, the results from BABAR LA-ISR analysis with the full data sets [12] are reviewed. 


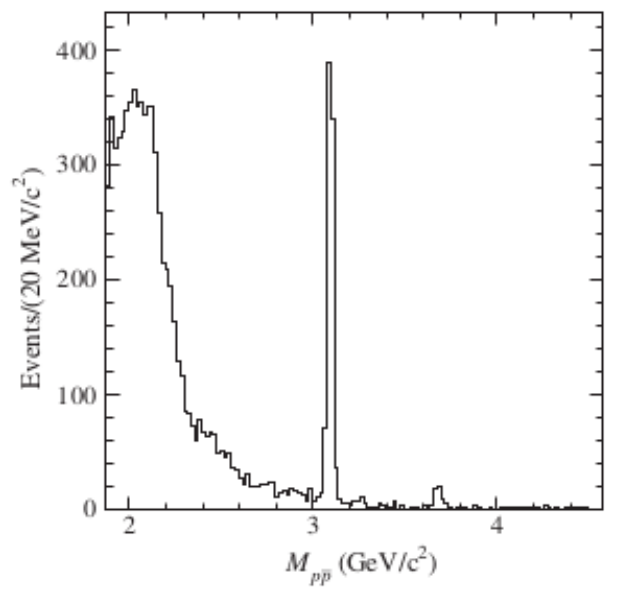

(a) $p \bar{p}$ invariant mass (BABAR LA-ISR)

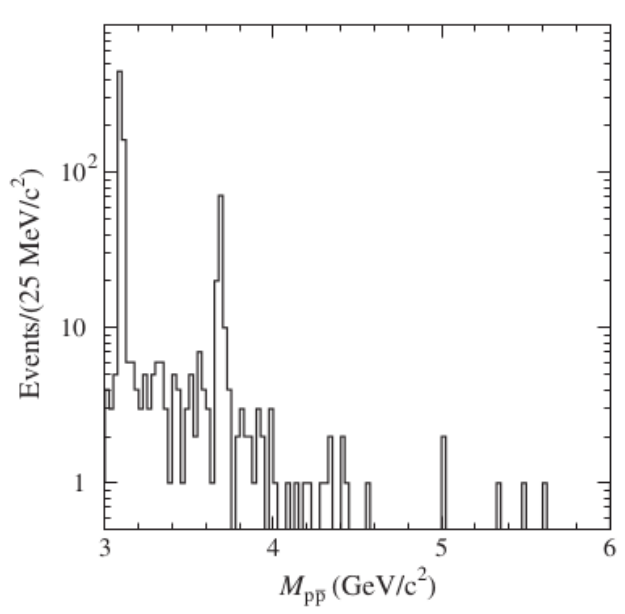

(b) $p \bar{p}$ invariant mass (BABAR SA-ISR)

Figure 3. The $p \bar{p}$ invariant mass spectrum from BABAR LA-ISR (a) and SA-ISR (b) analyses $[12,13]$.

Two ISR analyses (SA-ISR and LA-ISR) were performed at the BESIII experiment for the proton EMFFs measurements by using data sets collected at seven c.m. energies between 3.773 and $4.600 \mathrm{GeV}$ with a total integrated luminosity of $7.5 \mathrm{fb}^{-1}[16,17]$. Similar to the BABAR experiment, both analyses required that two charged tracks, generated from the interaction region, are identified as a proton and an antiproton. For the LAISR analysis, an additional most-energetic photon is included together with the proton and antiproton for a four-momentum kinematic fit to suppress the background. The dominant background is from the $e^{+} e^{-} \rightarrow p \bar{p} \pi^{0}$ process with one photon from the $\pi^{0}$ decay misidentified as the ISR photon. For the SA-ISR analysis, the ISR photon escapes through the beam pipes and consequently, requirements on the missing momentum and missing mass squared of the proton and antiporoton system are used to further suppress background such as two-photon, $p \bar{p} \pi^{0}$ and higher order ISR processes. Figure 4 shows the $p \bar{p}$ invariant mass spectra from BESIII LA-ISR (a) and SA-ISR (b) analyses.

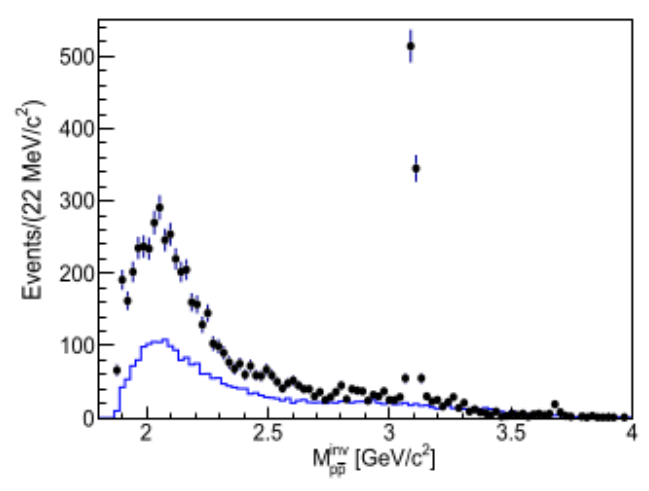

(a) $p \bar{p}$ invariant mass (BESIII LA-ISR)

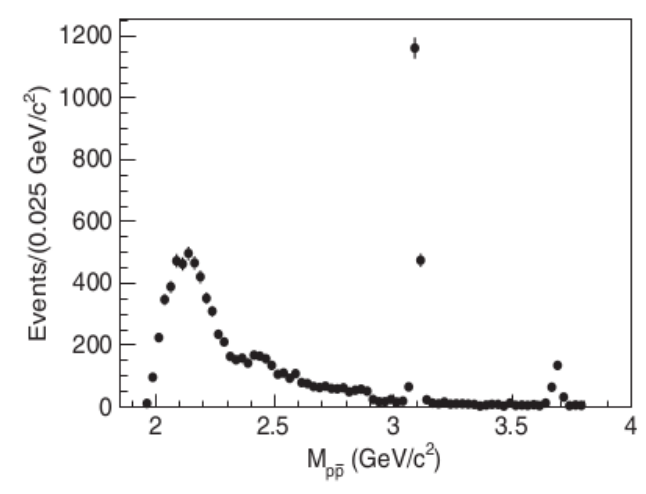

(b) $p \bar{p}$ invariant mass (BESIII SA-ISR)

Figure 4. The $p \bar{p}$ invariant mass spectrum from BESIII ISR analyses: (a) from LA-ISR analysis, the blue histogram represents the background events from $e^{+} e^{-} \rightarrow p \bar{p} \pi^{0}$ process [17], (b) from SA-ISR analysis [16].

The Born cross section of $e^{+} e^{-} \rightarrow p \bar{p}$, the proton effective form factor and the ratio of the proton EMFFs were measured at different $p \bar{p}$ invariant mass region for the LAand SA-ISR analyses from both BABAR and BESIII experiments, and Tab. 1 summarizes the information of the four analyses. 
Table 1: Information of the proton EMFFs measurements with LA- and SA-ISR analyses at BABAR and BESIII experiments.

\begin{tabular}{|c|c|c|c|c|c|}
\hline \multirow{2}{*}{ Experiment } & \multirow{2}{*}{$\begin{array}{c}\sqrt{s} \text { (range) } \\
{[\mathrm{GeV}]}\end{array}$} & \multirow{2}{*}{$\begin{array}{c}\mathcal{L}_{i n t} \\
{\left[\mathrm{fb}^{-1}\right]}\end{array}$} & \multicolumn{2}{|c|}{$\sqrt{q^{2}}$ range $\left[\mathrm{GeV} / \mathrm{c}^{2}\right]$} & \multirow{2}{*}{$\begin{array}{c}\text { total events } \\
\text { on } \sigma_{p \bar{p}}\end{array}$} \\
\hline & & & on $\sigma_{p \bar{p}}\left(G_{\text {eff }}\right)$ & on $R_{\mathrm{em}}$ & \\
\hline$\overline{\mathrm{BABA}}$ & \multirow{2}{*}{10.58} & \multirow{2}{*}{469} & $1.877-4.5$ & $1.877-3.0$ & $6876.0 \pm 107.2$ \\
\hline BABAI & & & $3.0-6.5$ & - & 13.2 \\
\hline BESIII LA-ISR & \multirow{2}{*}{$3.773-4.60$} & \multirow{2}{*}{7.5} & \multicolumn{2}{|c|}{$1.876-3.0$} & 2952. \\
\hline BESIII SA-ISR & & & $2.0-3.8$ & $2.0-3.0$ & $6714.2 \pm 87.4$ \\
\hline
\end{tabular}

\subsection{Born cross section of $e^{+} e^{-} \rightarrow p \bar{p}$ and proton effective $F F$}

The Born cross section of $e^{+} e^{-} \rightarrow p \bar{p}$ is calculated in small $p \bar{p}$ invariant mass intervals,

$$
\sigma_{p \bar{p}}^{i}\left(m_{p \bar{p}}\right)=\frac{\mathcal{N}_{p \bar{p}}^{i}}{\epsilon^{i}\left(1+\delta^{i}\right) \mathcal{L}^{i}},
$$

where the superscript $i$ represents the ${ }^{i}$ th $p \bar{p}$ mass interval, $\mathcal{N}_{p \bar{p}}^{i}$ is the number of $p \bar{p}$ events, $\epsilon^{i}$ and $\left(1+\delta^{i}\right)$ are the detection efficiency and the radiative correction factor determined by the MC simulation, and $\mathcal{L}^{i}$ is the ISR differential luminosity calculated as,

$$
\mathcal{L}^{i}=\int W(s, x) \mathcal{L} d x, \quad x=1-\frac{q^{2}}{s},
$$

where $q^{2}$ is the four momentum transfer squared for the ${ }^{i}$ th $p \bar{p}$ mass interval. In BABAR LA-ISR analysis, the process of $e^{+} e^{-} \rightarrow p \bar{p} \gamma$ is simulated to extract the selection efficiency and radiative correction factor at the range: $20^{\circ}<\theta_{\gamma}^{*}<160^{\circ}$ in the $e^{+} e^{-}$c.m. frame, therefore a reduced phase-space factor $\cos \theta_{\gamma}^{0}$ with $\theta_{\gamma}^{0}=20^{\circ}$ was included to calculate the differential luminosity by using the equation $6[11,12]$. In both analyses from the BESIII experiment, the full radiative function $W(s, x)$ (Eq. 7) was used for the differential luminosity calculation, since the process was simulated in a full phase space $[16,17]$. The analyses from BESIII experiment contain data sets from seven c.m. energies with different integrated luminosity, the differential luminosity was calculated from each data set and summed up together. To determine the MC efficiency and the radiative correction factor, their mean value was calculated from all the MC simulations from seven c.m. energies.

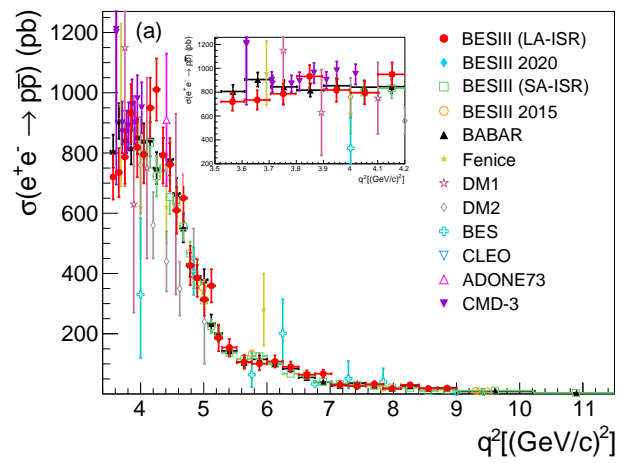

(a) cross section of $e^{+} e^{-} \rightarrow p \bar{p}$

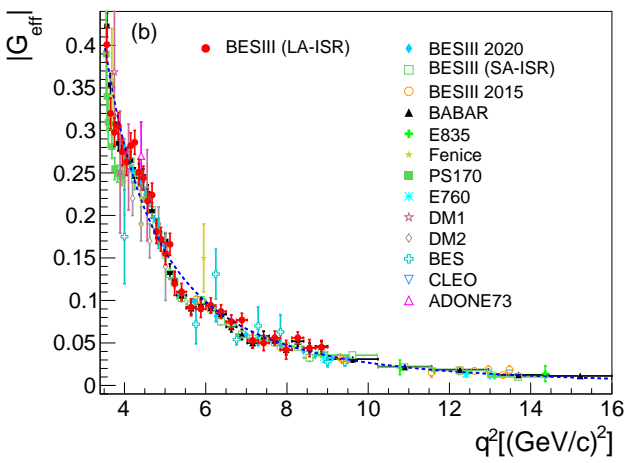

(b) effective FF of the proton

Figure 5. The cross section for the process $e^{+} e^{-} \rightarrow p \bar{p}$ (a) and the effective FF of the proton (b) [17]. The experimental data presented also include measurements from direct annihilation process.

The effective form factor of the proton is extracted from the $e^{+} e^{-} \rightarrow p \bar{p}$ cross section measurements for both LA- and SA-ISR analyses at BABAR and BESIII experiments according to Eq. 2 and 3. Figure 5 shows the results for the $e^{+} e^{-} \rightarrow p \bar{p}$ cross section (Fig. 
5 (a)) and the proton effective FF (Fig. 5 (b)) from all the analyses with the ISR process at BABAR and BESIII experiments, together with other available experimental data from direct annihilation process.

\subsection{The ratio of the proton FFs from the ISR process}

Taking advantage of the strong boost of the ISR photon, a full angular distribution of the proton can be obtained from the $e^{+} e^{-} \rightarrow p \bar{p} \gamma$ process. This is in contrast to most analyses using the direct annihilation process, which have to limit the angular range due to detector acceptance. Therefore, the ISR method allows to extract the ratio of the proton FFs with a relative higher precision comparing to direct annihilation measurements with comparable statistics. Both LA-ISR analyses from BABAR and BESIII measured the ratio of the proton FFs at the $p \bar{p}$ invariant mass region of threshold $-3.0 \mathrm{GeV} / \mathrm{c}^{2}$ for six mass intervals, while the BESIII SA-ISR analysis measured the ratio between $2.0-3.0 \mathrm{GeV} / \mathrm{c}^{2}$ for three mass intervals with reduced proton angular distributions. Figure 6 shows examples of proton angular distributions including a fit to extract the ratio of the proton FFs $[12,16,17]$.

The results of the extracted ratio of the proton FFs from the three measurements are shown in Fig. 7, including measurements from direct annihilation processes. Compared to the Born cross section measurements, the uncertainty both in terms of $R_{\mathrm{em}}(\mathrm{y}$ axis) and $\sqrt{q^{2}}$ (x axis) is till very large due to limited statistics. The results of the proton FFs ratio are consistent for measurements (direct annihilation and ISR processes) from the $e^{+} e^{-}$ collider experiments. At the region close to the $p \bar{p}$ threshold $\left(q^{2}<4.2(\mathrm{GeV} / \mathrm{c})^{2}\right)$, recent results of the ratio obtained with ISR process at BABAR and BESIII, as well as by the most precise measurement with the direct annihilation process at BESIII, disfavor of the early measurement from the PS170 experiment with $p \bar{p} \rightarrow e^{+} e^{-}$annihilation process.

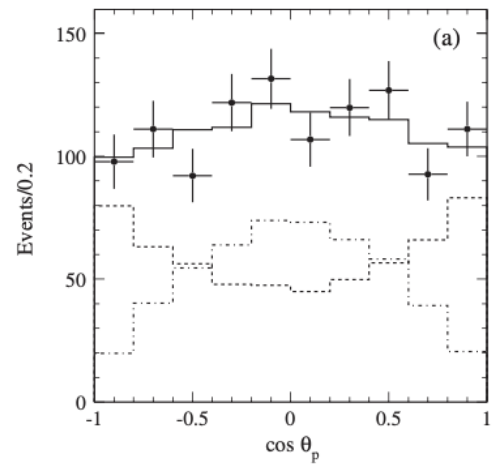

(a) BABAR LA-ISR

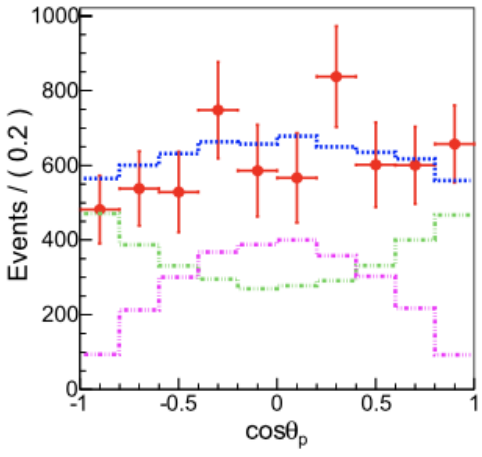

(b) BESIII LA-ISR

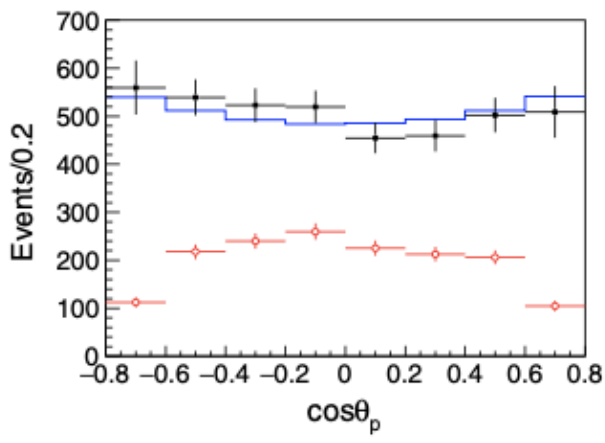

(c) BESIII SA-ISR

Figure 6. Examples of proton angular distributions including a fit to extract the ratio of the proton FFs from the BABAR (a) and BESIII (b) LA-ISA analyses (threshold- $1.95 \mathrm{GeV} / \mathrm{c}^{2}$ ), and the BESIII SA-ISR (c) analysis $\left(2.3-2.6 \mathrm{GeV} / \mathrm{c}^{2}\right)[12,16,17]$. 


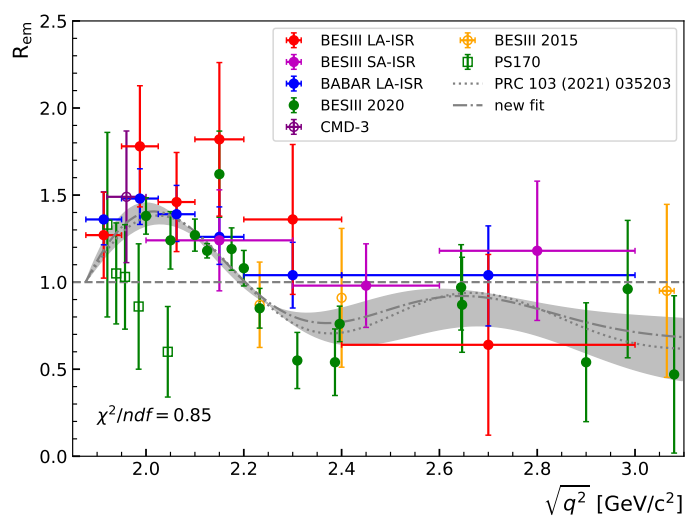

Figure 7. The ratio of the proton FFs from BABAR and BESIII LA-ISR analyses at the $p \bar{p}$ invariant mass region of threshold-3.0 GeV/ $\mathrm{c}^{2}$, and from BESIII SA-ISR analysis at the mass region of 2.0-3.0 GeV/ $/ \mathrm{c}^{2}$. Results also include measurements from PS170, BESIII (direct annihilation) and CMD-3 experiments [47-49]. The dotted line is the fit result in Ref. [46], the dot-dashed line shows a new fit result with the same formula by including both BESIII ISR results, and the grey band shows the standard deviation of this fit.

\section{Discussions and conclusions}

\subsection{Phenomenology studies on the proton EMFFs}

The QCD calculation of the nucleon EMFFs in the few GeV momentum transfer region, where non-perturbative methods are required, is complicated. Different models have been developed to describe the data in the space-like region but only some of them can be extended to the time-like region (for review see Refs. [37,38]), such as the models based on dispersion relations [39] and vector meson dominance [40,41]. This region has recently attracted a lot of attention, in particular after the precise ISR measurements from BABAR were available. The BABAR data on the $e^{+} e^{-} \rightarrow p \bar{p}$ Born cross section and on the effective EMFF show evidence of some structures (Fig. 5). These structures have been later confirmed by the 3 independent analyses from the BESIII collaboration based on ISR and energy scan techniques. Theoretical studies have attempted to reproduce these structures and to provide an explanation of their origin. In 2014, an improved parametrization of the nucleon EMFFs based on generalized vector dominance has been developed and implemented in the PHOKHARA event generator [36]. It describes well the measurements from BABAR, as well as the space-like data on the proton form factor ratio using polarized beam and/or target. In 2015, Lorenz et al. analyzed the EMFFs ratio and the effective FF of the proton from both TL and SL regions, and discussed possible contributions to the nucleon FFs from the vector meson $\phi(2170)$ or from final-state interaction (FSI) at the $N \bar{\Delta}+$ c.c and $\Delta \bar{\Delta}$ thresholds [42]. A recent phenomenology study, by Cao and Dai, has a joint discussion on the effective FF of proton and neutron, where the periodic structures could be explained by the contributions from pure isoscalar or isovector, or their orthogonal interference [43]. 


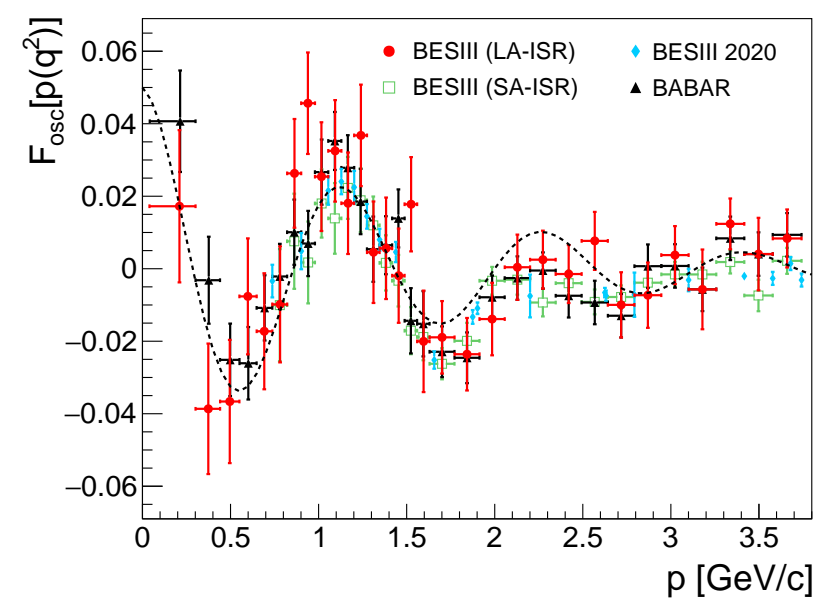

Figure 8. Periodic oscillation structure of the proton effective FF[17]. The dotted line represents the oscillation contribution, which is described by Eq. 12 and the value of fit parameters are taken from Ref. [45].

In 2015, Bianconi and Tomasi-Gustafsson studied the proton effective FF from BABAR LA-ISR measurement, and found the data can be best reproduced by a threepole function $\left(F_{3 p}(s)\right)$ with an additional periodic oscillation part $\left(F_{o s c}[p(s)]\right)[44,45]$. By including more experimental data from BESIII (direct annihilation and SA-ISR analyses) and CMD-3 experiments, this research was updated in 2021[46].

$$
\begin{gathered}
\left|F_{p}^{f i t}\left(q^{2}\right)\right|=F_{3 p}\left(q^{2}\right)+F_{o s c}\left[p\left(q^{2}\right)\right], \\
F_{3 p}\left(q^{2}\right)=\frac{F_{0}}{\left(1+q^{2} / m_{a}^{2}\right)\left(1-q^{2} / m_{0}^{2}\right)^{2}}, \\
F_{o s c}\left[p\left(q^{2}\right)\right]=A e^{-B p} \cos (C p+D),
\end{gathered}
$$

where $p\left(q^{2}\right)=\sqrt{q^{2}(\tau-1)}$ is the relative momentum of the proton in the frame where the antiproton is at rest, the standard dipole parameter $m_{0}^{2}=0.71(\mathrm{GeV} / \mathrm{c})^{2}$, and other parameters are extracted by fitting to the experimental data, the values can be found in the reference [46]. Figure 8 shows the periodic oscillation structure of the proton effective FF described by Eq. 12, in which the values of parameters are taken from Ref. [45].

Similar to the effective FF of the proton, a fit was performed in Ref. [46] on the ratio of the proton EMFFs with the function including a monopole decrease and a damped oscillation,

$$
\mathrm{R}_{\mathrm{em}}=\frac{1}{1+\left(q-2 m_{p}\right)^{2} / r_{0}}\left[1+r_{1} e^{-r_{2}\left(q-2 m_{p}\right)} \sin \left(r_{3}\left(q-2 m_{p}\right)\right)\right] .
$$

As shown in Fig. 7, the fit (dotted line), performed in Ref. [46], was only applied on the results from BABAR LA-ISA and BESIII energy scan (2020) measurements. By including more experimental results from both BESIII ISR measurements, a new fit (dot-dashed line in Fig. 7) is performed in this report, in which the result is slightly changed with an improved fitting quality. The grey shadow represents a one standard deviation band.

\subsection{Discussion of the proton EMFFs from the ISR process}

The ISR Process has advantages to investigate the proton EMFFs at the $e^{+} e^{-}$collider experiments.

So far, it is the only method used to extract the ratio of proton EMFFs at the $p \bar{p}$ threshold. Because of the very low momentum of the produced proton/anti-proton at the threshold from the direct annihilation process, it is not possible to reconstruct the 
charged tracks in a tracking system of the detector. The CMD-3 experiment has measured the cross section of $e^{+} e^{-} \rightarrow p \bar{p}$ at the invariant mass very close to the threshold (1.89 and $1.90 \mathrm{GeV} / \mathrm{c}^{2}$ ) by counting the annihilation of the antiproton in the beam pipe or in the drift chamber (tracking sub-detector) [49]. The ratio or the individual FF could not be extracted at these invariant mass regions because the angular distribution analysis is impossible without the proton/antiproton charged track reconstruction. With the strong boost of an ISR photon, the proton and antiproton are produced at their threshold with large momenta, therefore full information of the momentum and PID can be obtained for the charged tracks of the $p \bar{p}$ system, thus it is possible to extract the ratio of the proton EMFFs (or individual FFs) by analyzing the angular distribution of the proton at the $p \bar{p}$ threshold region.

Table 2: A simple comparison for the precision of the proton FFs ratio measured by the ISR process and the direct annihilation process from BABAR, BESIII and CMD-3 experiments. [Note: the uncertainty of the proton FFs ratio $\left(\Delta R_{e m} / R_{e m}\right)$ is statistical only, except the one from BESIII SA-ISR analysis.]

\begin{tabular}{|c|c|c|c|c|}
\hline$q[\mathrm{GeV} / \mathrm{c}]$ & $\mathrm{N}_{\mathrm{evt}}$ & $1 / \sqrt{\mathrm{N}_{\mathrm{evt}}}$ & $\Delta \mathrm{R}_{\mathrm{em}} / \mathrm{R}_{\mathrm{em}}$ & experiment \\
\hline $2.025-2.100$ & 1328 & $2.74 \%$ & $10.79 \%$ & BABAR LA-ISR [12] \\
\hline $2.025-2.100$ & 560 & $4.23 \%$ & $18.49 \%$ & BESIII LA-ISR [17] \\
\hline $2.0-2.3$ & 4283 & $1.53 \%$ & $\sim 23.39 \%$ & BESIII SA-ISA [16] \\
\hline 2.125 & 50312 & $0.45 \%$ & $3.39 \%$ & BESIII energy scan [48] \\
\hline $1.92-2.00$ & 2577 & $1.97 \%$ & $15.44 \%$ & CMD-3 (combined) [49] \\
\hline
\end{tabular}

A full angular distribution of the proton improves the precision of the ratio of the proton FFs. It is not possible to have a full angular distribution of the proton from the direct annihilation process due the non- $4 \pi$ acceptance of a detector. As another benefit of the boost of the ISR photon, a full angular distribution of the proton (Fig. 6) is obtained in the $p \bar{p}$ c.m. frame, and the ratio is extracted with higher precision by fitting to the full angular distribution. Table 2 lists a simple comparison for the precision of the proton FFs ratio, which were measured by the LA-ISR analyses from BABAR and BESIII, the SA-ISR analysis from BESIII, and the direct annihilation analyses from BESIII and CMD-3 experiments. The impact of the full angular distribution can be seen by comparing the statsitical uncertainty on the proton FFs ratio to the corresponding one of the total number of observed events.

In addition, the ISR method provides continuous measurements of the proton EMFFs in a large $p \bar{p}$ invariant mass range. The combined BABAR and BESIII ISR measurements of the Born cross section and the proton effective FF cover the range from the threshold up to $6.5 \mathrm{GeV} / \mathrm{c}^{2}$, while the existing data from scan experiments are only available at discrete $p \bar{p}$ invariant mass points below $\sim 4.2 \mathrm{GeV} / \mathrm{c}^{2}$.

\subsection{Expectation of the nucleon EMFFs measurements through ISR technique}

The disadvantage of the ISR process for the proton EMFFs measurement is obviously that the statistics is much lower compared to the direct annihilation process, therefore very high luminosity is necessary to compensate. The Belle-II experiment on the $B$ factory SuperKEKB is starting data collection and will be able to contribute for the proton EMFFs study in the future. The Belle-II experiment has the goal to obtain a total integrated luminosity of $50 \mathrm{ab}^{-1}$ in the c.m. energy range from 9.46 to $11.24 \mathrm{GeV}$, and it has planned to collect $\sim 5 \mathrm{ab}^{-1}$ integrated luminosity in 2020 [50]. According to a recent report, the plan is delayed and an integrated luminosity of $231 \mathrm{fb}^{-1}$ was accumulated until 2021 [51]. The BESIII experiment is continuing to collect data between $2.00-4.95 \mathrm{GeV}$, where many large data sets might be helpful to improve measurements of the proton EMFFs in a large $q^{2}$ range, and it is even possible to access other baryonantibaryon channels. The BESIII experiment has accumulated large luminosity data sets above $3.770 \mathrm{GeV}$. The data sets, which can be used for the baryon EMFFs measurements 
with ISR technique, are estimated to be $\sim 22 \mathrm{fb}^{-1}$ until to 2021 [52]. In next two years, BESIII will collect data at $\sqrt{s}=3.773 \mathrm{GeV}$ to obtain a total luminosity up to $20 \mathrm{fb}^{-1}$ (including the $2.9 \mathrm{fb}^{-1}$ from previous data) $[53,54]$. Considering that one disadvantage of the ISR technique is worse resolution of the $p \bar{p}$ invariant mass, BESIII experiment also plans to explore the nucleon production threshold region through direct annihilation process by taking data below $2.0 \mathrm{GeV}$ [55].

Regarding the expected luminosities from both experiments in the next few years, researches for the EMFFs of the proton may achieve significant progress with the ISR technique, especially for the EMFFs ratio of the proton close to the $p \bar{p}$ threshold region, where the detection efficiency is almost zero for the low momentum proton/antiproton tracks produced from the direct annihilation process.

\subsection{Summary}

The ISR technique to analyze proton EMFFs is not only a complementary method, but also has the advantages to access the proton-antiproton threshold and to improve the precision of the ratio measurement with full proton angular distribution analysis. The BABAR experiment started the proton EMFFs research with the ISR technique, and the results of the proton EMFFs ratio show a tension on the ratio of the proton EMFFs to the results from previous experiment PS170. BESIII experiment performed the proton EMFFs measurements through the direct annihilation and ISR processes with different data sets, very high precision for the measurements on the $e^{+} e^{-} \rightarrow p \bar{p}$ cross section and the FFs (ratio) is achieved from the direct annihilation analysis, and large invariant mass range from the $p \bar{p}$-threshold to $3.8 \mathrm{GeV} / \mathrm{c}^{2}$ is covered from the ISR analyses. Future measurements with higher precision, in particular close to the $p \bar{p}$ threshold, will enhance our knowledge on the proton EMFFs in the time-like region.

Author Contributions: All authors have read and agreed to the published version of the manuscript.

Funding: This work is supported in part by the 100 Talents Program of CAS; the Key Research Program of CAS under grant No. XDB34030301, and ERC under contract No. 758462; European Union Horizon 2020 research and innovation programme under contract No. Marie ShlodowskaCurie grant agreement No. 645664, 872901 and 894790; German Research Foundation DFG under contract No. 443159800 and Collaborative Research Center CRC 1044.

Institutional Review Board Statement: Not applicable

Informed Consent Statement: Not applicable

Data Availability Statement: The original data are made publicly available by the referred collaborations.

Acknowledgments: Authors would like to thank the editors of the special issue of the journal, Dr. Monica Bertani, Prof. Simone Pacetti and Dr. Alessio Mangoni for the organization and helps. Authors would like to thank Prof. Guangshun Huang for organizing and coordinating the papers for the special issue, and thank Dr. Christoph Rosner and Dr. Lei Xia for their careful reading and valuable suggestions for this paper.

Conflicts of Interest: The authors declare no conflict of interest.

\section{References}

1. Baier, V. N.; Khoze, V. A. Photon emission in muon pair production in electron-positron collisions. Sov. Phys. JETP 1965, 21, 629-632.

2. Baier, V. N.; Khoze, V. A. Radiation accompanying two particle annihilation of an electron-positron pair. Sov. Phys. JETP 1965, 21, 1145-1150.

3. Arbuzov, A. B. et al. Hadronic cross-sections in electron - positron annihilation with tagged photon. JHEP 1998, $12,009$.

4. Benayoun, M. et al. Spectroscopy at B factories using hard photon emission. Mod. Phys. Lett. A 1999, 14, 2605-2614.

5. Binner, S. Kuhn, J. H.; Melnikov, K. Measuring $\sigma\left(e^{+} e^{-} \rightarrow\right.$ hadrons $)$ using tagged photon. Phys. Lett. B 1999, $459,279-287$.

6. Konchatnij, M. I.; Merenkov, N. P. Scanning of hadron cross-section at DAPHNE by analysis of initial state radiative events. JETP Lett. 1999,69, 811-818. 
7. "Rodrigo, G. et al. NLO QED corrections to ISR in $e^{+} e^{-}$annihilation and the measurement of $\sigma\left(e^{+} e^{-} \rightarrow\right.$ hadrons $)$ using tagged photons. Eur. Phys. J. C 2001, 22, 81-88.

8. Rodrigo, G. et al. Radiative return at NLO and the measurement of the hadronic cross-section in electron positron annihilation. Eur. Phys. J. C 24, 2002, 71-82.

9. Czyz, H.; Kuhn, Johann H. Four pion final states with tagged photons at electron positron colliders. Eur. Phys. J. C 18, 2001, 497-509.

10. Aubert, B. et al. (BABAR Collaboration), The BABAR detector. Nucl. Inst. Meth. Phys. Res. A 2002, 479, 1-116.

11. Aubert, B. et al. (BABAR Collaboration), Stuty of $e^{+} e^{-} \rightarrow p \bar{p}$ using initial state radiation with BABAR. Phys. Rev. D 2006, 73, 012005.

12. Lees, J. P. et al. (BABAR Collaboration), Stuty of $e^{+} e^{-} \rightarrow p \bar{p}$ via initial-state radiation at BABAR. Phys. Rev. D 2013, 87, 092005.

13. Lees, J. P. et al. (BABAR Collaboration), Measurement of $e^{+} e^{-} \rightarrow p \bar{p}$ cross section in the energy range from 3.0 to $6.5 \mathrm{GeV}$. Phys. Rev. D 2013, 88, 072009.

14. Aubert, B. et al. (BABAR Collaboration), Study of $e^{+} e^{-} \rightarrow \Lambda \bar{\Lambda}, \Lambda \bar{\Sigma}^{0}, \Sigma^{0} \bar{\Sigma}^{0}$ using initial state radiation with BABAR. Phys. Rev. D 2007, 76, 092006.

15. Pakhlova, G. et al. (Belle Collaboration) Observation of a near-threshold enhancement in the $e^{+} e^{-} \rightarrow \Lambda_{c} \bar{\Lambda}_{c}$ cross section using initial-state radiation. Phys. Rev. Lett. 2008, 101, 172001.

16. Ablikim, M. et al. (BESIII Collaboration), Study of the process $e^{+} e^{-} \rightarrow p \bar{p}$ via initial state radiation at BESIII. Phys. Rev. D 2019, 99, 092002.

17. Ablikim, M. et al. (BESIII Collaboration), Measurement of proton electromagnetic form factors in the time-like region using initial state radiation at BESIII. Phys. Lett. B 2021, 817, 136328.

18. Aubert, B. et al. (BABAR Collaboration), Precise measurement of the $e^{+} e^{-} \rightarrow \pi^{+} \pi^{-}(\gamma)$ cross section with the initial state radiation method at BABAR. Phys. Rev. Lett. 2009, 103, 231801.

19. Lees, J. P. et al. (BABAR Collaboration), Precision measurement of the $e^{+} e^{-} \rightarrow K^{+} K^{-}(\gamma)$ cross section with the initial-state radiation method at BABAR. Phys. Rev. D 2013, 88, 032013.

20. Lees, J. P. et al. (BABAR Collaboration), Study of the $e^{+} e^{-} \rightarrow K^{+} K^{-}$reaction in the energy range from 2.6 to $8.0 \mathrm{GeV}$. Phys. Rev. D 2015, 92, 072008.

21. Aloisio, A. et al. (KLOE Collaboration), Measurement of $\sigma\left(e^{+} e^{-} \rightarrow \pi^{+} \pi^{-} \gamma\right)$ and extraction of $\sigma\left(e^{+} e^{-} \rightarrow \pi^{+} \pi^{-}\right)$below $1-\mathrm{GeV}$ with the KLOE detector. Phys. Lett. B 2005, 606, 12-24.

22. Ambrosino, F. et al. (KLOE Collaboration), Measurement of $\sigma\left(e^{+} e^{-} \rightarrow \pi^{+} \pi^{-} \gamma(\gamma)\right.$ and the dipion contribution to the muon anomaly with the KLOE detector. Phys. Lett. B 2009, 670, 285-291.

23. Babusci, D. et al. (KLOE Collaboration), Precision measurement of $\sigma\left(e^{+} e^{-} \rightarrow \pi^{+} \pi^{-} \gamma\right) / \sigma\left(e^{+} e^{-} \rightarrow \mu^{+} \mu^{-} \gamma\right)$ and determination of the $\pi^{+} \pi^{-}$contribution to the muon anomaly with the KLOE detector. Phys. Lett. B 2013, 720, 336-343.

24. Ambrosino, F. et al. (KLOE Collaboration), Measurement of $\sigma\left(e^{+} e^{-} \rightarrow \pi^{+} \pi^{-}\right)$from threshold to $0.85 \mathrm{GeV}^{2}$ using Initial State Radiation with the KLOE detector. Phys. Lett. B 2011, 700, 102-110.

25. Ablikim, M. et al. (BESIII Collaboration), Measurement of the $e^{+} e^{-} \rightarrow \pi^{+} \pi^{-}$cross section between 600 and $900 \mathrm{MeV}$ using initial state radiation. Phys. Lett. B 2016, 753, 629-638. [Erratum: Phys.Lett.B 2021, 812, 135982.]

26. Zichichi, A. et al. Proton anti-proton annihilation into electrons, muons and vector bosons. Nuovo Cim. 1962, 24, 170-180.

27. Baldini Ferroli, R.; Pacetti, S.; Zallo, A. No Sommerfeld resummation factor in $e^{+} e^{-} \rightarrow p \bar{p}$ ? Eur. Phys. J. A 2012, $48,33$.

28. Bonneau, G.; Martin, F. Hard photon emission in $e^{+} e^{-}$reactions. Nucl. Phys. B 1971, 27, 381.

29. Druzhinin, V. P. et al. Hadron Production via $e^{+} e^{-}$Collisions with Initial State Radiation. Rev. Mod. Phys. 2011, $83,1545$.

30. Czyz, H. et al. Nucleon form-factors, B meson factories and the radiative return. Eur. Phys. J. C 2004, 35, 527-536.

31. Caffo, M.; Czyz, H.; Remiddi, E. BHAGEN95: A Monte Carlo program for Bhabha scattering at LEP-1 / SLC and LEP-2 energies. Nuovo Cim. A 1997, 110, 515-536.

32. Caffo, M.; Czyz, H.; Remiddi, E. Order- $\alpha^{2}$ leading logarithmic corrections in Bhabha scattering at LEP / SLC energies. Phys. Lett. B 1994, 327, 369-376.

33. Kuhn, J. H.; Rodrigo, G. The Radiative return at small angles: Virtual corrections. Eur. Phys. J. C 2002, 25, $215-222$.

34. Czyz, H. it al. The Radiative return at $\phi$ - and B-factories: Small angle photon emission at next-to-leading order. Eur. Phys. J. C 2003, 27, 563-575.

35. Czyz, H. it al. The Radiative return at $\phi$ - and B-factories: FSR for muon pair production at next-to-leading order. Eur. Phys. J. C 2005, 39, 411-420.

36. Czyz, H.; Kuehn, J. H.; Tracz, S. Nucleon form factors and final state radiative corrections to $e^{+} e^{-} \rightarrow p \bar{p} \gamma$. Phys. Rev. D 2014, 38, 114021.

37. Denig, A.; Salmè, G. Nucleon electromagnetic form factors in the timelike region. Prog. Part. Nucl. Phys. 2013, 68, $113-157$.

38. Pacetti, S.; Baldini Ferroli, R.; Tomasi-Gustafsson, E. Proton electromagnetic form factors: Basic notions, present achievements and future perspectives. Phys. Rep. 2015, 550-551, 1-103.

39. Belushkin, M. A.; Hammer, H.-W.; Meißner, Ulf-G. Dispersion analysis of the nucleon form factors including meson continua. Phys. Rev. C 2007, 75, 035202.

40. Bijker, R.; Iachello, F. Reanalysis of the nucleon spacelike and timelike electromagnetic form factors in a two-component model. Phys. Rev. C 2004, 69, 068201. 
41. Lomon, E. L.; Pacetti, S. Timelike and spacelike electromagnetic form factors of nucleons, a unified description. Phys. Rev. D 2012, 85, 113004. [Erratum: Phys. Rev. D 2012, 86, 039901.]

42. Lorenz, I. T.; Hammer, H.-W.; Meißner, Ulf-G. New structure in the proton-antiproton system. Phys. Rev. D 2015, $92,034018$.

43. Cao, X.; Dai, J. P. Timelike nucleon electromagnetic form factors: All about interference of isospin amplitudes. 2021, arXiv:2109.15132.

44. Bianconi, A.; Tomasi-Gustafsson, E. Periodic interference structures in the timelike proton form factor. Phys. Rev. Lett. 2015, 114, 232301.

45. Bianconi, A.; Tomasi-Gustafsson, E. Phenomenological analysis of near-threshold periodic modulations of the proton timelike form factor. Phys. Rev. C 2016, 93, 035201.

46. Tomasi-Gustafsson, E.; Bianconi, A.; Pacetti, S. New fit of timelike proton electromagnetic formfactors from $e^{+} e^{-}$colliders. Phys. Rev. C 2021, 103, 035203.

47. Carlin, R. et al. (PS170 Collaboration), The proton electromagnetic form factor $\left(p \bar{p} \rightarrow e^{+} e^{-}\right)$. Nucl. Phys. B 1989, 8, $203-208$.

48. Ablikim, M. et al. (BESIII Collaboration), Measurement of Proton Electromagnetic Form Factor in $e^{+} e^{-} \rightarrow p \bar{p}$ in the Energy retion 2.00-3.08 GeV. Phys. Rev.Lett. 2020, 124, 042001.

49. Akhmetshin, R. R. et al. Study of the process $e^{+} e^{-} \rightarrow p \bar{p}$ in the c.m. energy range form threshold to $2 \mathrm{GeV}$ with the CMD-3 detector. Phys. Lett. B 2016, 759, 634-640.

50. Kou, E. et al. (Belle-II Collaboration) The Belle II Physics Book. Prog. Theor. Exp. Phys. 2019, 123C01, 1-654.

51. Onuki, Y. Belle II: status and prospects, Presentation on QCD21 - 24th High Energy Physcis International Conference, July 5-9, 2021.

52. The BESIII Experiment. Available online: User Information - Data Sets.

53. Asner, D. M. et al. (BESIII Collaboration), Physics at BESIII. Int. J. Mod. Phys. A 2009, 24, Issue: 1 supp.

54. Ablikim, M. et al. (BESIII Collaboration), Future Physics Programme of BESIII. Chin. Phys. C 2020, 44, 040001.

55. Huang, G. S.; Baldini Ferroli, R. Probing the internal structure of baryons. National Science Review 2021, nwab187. 